\title{
Medical management of Crohn's disease: state of the art and future perspectives
}

\author{
Fabio Salvatore Macaluso \\ Department of Medicine, Villa Sofia-Cervello Hospital, Palermo, Italy
}

\begin{abstract}
Over the past decade, the improvement in the understanding of the molecular mechanisms of Crohn's disease (CD) led to the development of more targeted therapies, including biologics - i.e. monoclonal antibodies that selectively block key mediators of inflammation - and novel small molecule drugs - i.e. compounds with a molecular weight $<1 \mathrm{kDa}$ able to diffuse through cell membranes and then fit for the oral route of administration - which will enrich the therapeutic armamentarium of CD soon. In parallel with the expansion of the medical options, the therapeutic targets to be achieved in patients with CD have changed. In particular, we moved from the simple control of symptoms to more ambitious goals which aim to permanently extinguish the inflammation, even the subclinical one. As a consequence, the role of some of the conventional drugs which have been used in $\mathrm{CD}$ for several years, such as 5-aminosalicylates and conventional immunosuppressants, is becoming more limited in favor of these new drugs. This profound modification of CD therapy and the intrinsic complexity of the disease are relevant to the point that the management of inflammatory bowel diseases is gradually becoming a subspecialty in the field of gastroenterology or internal medicine.
\end{abstract}

\section{Introduction}

Crohn's disease $(\mathrm{CD})$ is a progressive and potentially disabling condition that often requires lifelong medical management, as currently the precise etiology is unknown and therefore a causal therapy does not exist. ${ }^{1}$ The disease has periods of clinical remission alternated with recurrences. Unfortunately, there is not an exact matching between the symptoms of the patients and the real inflammatory activity of the disease.

Correspondence: Fabio Salvatore Macaluso, Department of Medicine, Villa Sofia-Cervello Hospital, 90146 Palermo, Italy.

Tel.: +39.091.6804752 - Fax: +39.091.6808225.

E-mail: fsmacaluso@gmail.com

Key words: Biologics; clinical guidelines; randomized controlled trials; small molecule drugs.

Conflict of interests: Fabio Salvatore Macaluso served as an advisory board member and/or received lecture grants from MSD, AbbVie, Biogen, and Takeda Pharmaceuticals. This paper was not funded.

Received for publication: 24 June 2019.

Accepted for publication: 1 July 2019.

This work is licensed under a Creative Commons Attribution NonCommercial 4.0 License (CC BY-NC 4.0).

${ }^{\circ}$ Copyright: the Author(s), 2019

Licensee PAGEPress, Italy

Italian Journal of Medicine 2019; 13:152-160

doi:10.4081/itjm.2019.1194
As a consequence, the subclinical inflammation that often persists during clinical remission may lead to the typical complications of the disease - strictures, fistulas and abscesses - and ultimately to progressive bowel damage and need for surgery, which is required at least once in half of the patients within 10 years of diagnosis. ${ }^{2}$ Furthermore, a third of patients will need repeated surgery, with a consequent risk of short bowel and/or a permanent stoma. ${ }^{3}$ Anyway, a huge improvement in the understanding of the molecular mechanisms of inflammatory bowel diseases (IBD) has been achieved over the past decade. ${ }^{4}$ The increasing knowledge of the immunological basis of the disease led to the introduction of more targeted therapies, namely biologics - i.e. monoclonal antibodies that selectively block key mediators of inflammation - and novel small molecule drugs - i.e. compounds with a molecular weight $<1 \mathrm{kDa}$ able to diffuse through cell membranes and then fit for the oral route of administration $^{5}$ - which will enrich the therapeutic armamentarium of $\mathrm{CD}$ soon. ${ }^{6}$

In parallel with the expansion of the medical options that is being observed in recent years and that hopefully - should lead to an improvement of the clinical outcomes, the therapeutic targets to be achieved in patients with $C D$ have changed. In particular, we moved from the simple control of symptoms to more ambitious goals which aim to permanently extinguish the inflammation, even the subclinical one. As a consequence, the role of some of the conventional drugs which have been used in CD for several years is becoming more limited in favor of these new drugs. This profound modification of $\mathrm{CD}$ therapy, coupled with 
the intrinsic complexity of the disease - patients with $\mathrm{CD}$ are different from one another - are relevant to the point that IBD management is gradually becoming a subspecialty in the field of gastroenterology or internal medicine.

\section{Therapeutic strategies and treatment targets}

Two main therapeutic approaches for the management of CD are classically described (Figure 1). The first one is based on the initial use of less powerful and safer - at least theoretically - therapies, with subsequent escalation to more potent treatments in the event of non-response. This is the so-called step-up approach, which should be correctly performed without lingering too much on a treatment line in the event of a non-response, passing quickly from one line to another (accelerated step-up). The second approach, defined as early top-down, is based on the use of the most effective therapies - especially biologics, alone or in combination with immunosuppressants - since the earliest stages of the disease, in order to prevent the bowel damage and disability. ${ }^{7}$ Both these approaches should be re-appraised today considering that novel treatment targets have been recently identified as the keys able to modify the natural course of the disease. In particular, as clinical symptoms are not uniformly matched with the real underlying inflammation, effective disease control can be reached only through treatments aiming beyond symptoms. In this line, mucosal healing - usually defined as the absence of deep ulcerations - has emerged as an important therapeutic target in IBD disease because it is associated with reduced recurrences of the disease and need for surgery. ${ }^{8}$ Similarly, the so-called deep remission (i.e. biochemical, clinical and endoscopic remission) has emerged as another relevant treatment target. ${ }^{9}$ Given these new goals, a top-down approach should be considered in patients with $\mathrm{CD}$ and unfavorable prognostic factors or severe disease. ${ }^{10}$ For the remaining patients, an accelerated step-up approach is reasonable. Nowadays, the treatment escalation of the stepup approach can be guided by close non-invasive monitoring of the disease using repeated assessments of fecal calprotectin and inflammatory biomarkers, in particular the C-reactive protein, which have been shown a good correlation with the endoscopic activity. ${ }^{11}$ This treat-to-target approach is based on the results of the recent CALM study which showed that, in patients with early $\mathrm{CD}$, timely escalations (and deescalation) with adalimumab based on fecal calprotectin and C-reactive protein values resulted in better clinical and endoscopic outcomes compared with escalations guided only by the symptoms. ${ }^{12}$

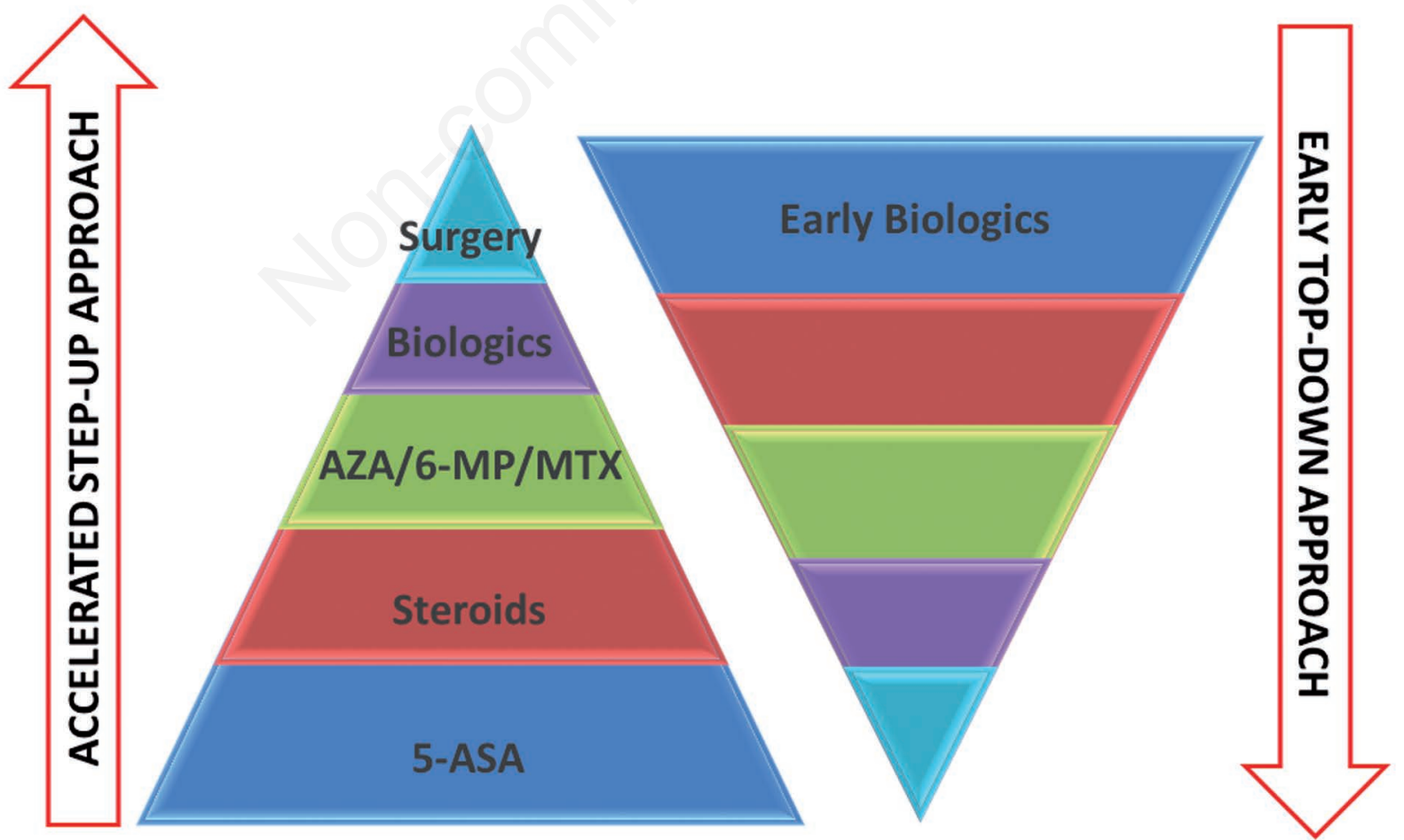

Figure 1. A schematic overview of the accelerated step-up vs top-down approaches in Crohn's disease. 5-ASA, 5-aminosalicylates; 6-MP, 6-mercaptopurine; AZA, azathioprine; MTX, methotrexate. 


\section{Therapeutic options for Crohn's disease}

CD includes a wide spectrum of clinical manifestations, whose management requires a specific therapeutic approach that should always be discussed with the patient. Generally speaking, medical therapy involves two temporal phases, namely the induction and the maintenance of remission. Practice guidelines ${ }^{13}$ can certainly be helpful for driving the therapeutic decisions, even if several recommendations are not evidence-based, but based on expert opinions. Patients are classified based on the extent of the disease, the degree of activity (mild, moderate and severe), the prevalent clinical manifestations (inflammatory/stricturing/fistulizing pattern) and the previous response to treatments. The specific clinical condition must therefore guide the choice of the therapeutic approach for each individual patient.

\section{5-aminosalicylates}

Mesalazine has been used for the treatment of CD for a long time, particularly for the induction of mildly active localized $\mathrm{CD}$ and for the maintenance of remission, due to evidence of efficacy derived from old trials. ${ }^{14,15}$ Furthermore, sulfasalazine 3-6 g/day resulted to be effective in colonic disease, but not in small bowel disease. ${ }^{16}$ Anyway, extensive recent systematic reviews of literature data have repeatedly shown no clinically relevant improvement with 5 -aminosalicylates. ${ }^{17}$ Furthermore, 5 -aminosalicylates resulted to be inferior to budesonide in a randomized clinical trial (RCT) ${ }^{18}$ As a consequence, 5-aminosalicylates are not recommended anymore for induction or maintenance of remission in $\mathrm{CD}$, while no treatment could be an option for a proportion of patients with very mild disease. Anyway, caution is needed in asserting with certainty the lack of efficacy of 5-aminosalicylates in CD, as most of the evidence comes from old studies, whose overall quality is generally low. Furthermore, 5aminosalicylates may be slightly effective to prevent the postoperative recurrence. ${ }^{19}$

\section{Corticosteroids}

Corticosteroids are still the mainstay for the induction of remission..$^{13}$ In particular, in case of localized ileal or ileocecal disease, budesonide is able to reach these sites, and thus should be preferred to systemic steroids due to its limited side effects. ${ }^{20}$ Systemic steroids (oral prednisone for moderately active disease, or intravenous prednisolone for severely active disease) should be used for all other locations. The optimal dose of systemic corticosteroids is approximately $0.75 \mathrm{mg} / \mathrm{kg} /$ day of prednisone (or equivalent dose of another steroid). Patients who do not respond to an adequate dose steroid therapy for more than two weeks should be considered as steroid-resistant, being candidates for a treatment with a biologic agent. Furthermore, it should be emphasized that a relevant proportion of patients become steroid dependent, ${ }^{21}$ and that steroids should never be used as maintenance therapy, because they are not effective for maintaining remission, and the adverse events related to their prolonged exposure are well-known, insidious, and dangerous. As a consequence, the use of steroids must always be accompanied by a strategy of correct tapering and by the link with a steroid-sparing strategy for the subsequent maintenance of remission.

\section{Immunosuppressants}

Immunosuppressants, particularly thiopurines (azathioprine at a dose of $2.5-3 \mathrm{mg} / \mathrm{kg} / \mathrm{day}$ or 6 -mercaptopurine at a dose of $1-1.5 \mathrm{mg} / \mathrm{kg} / \mathrm{day}$ ) are still frequently used for the treatment of $\mathrm{CD}$, particularly for the maintenance of remission in patients with steroiddependent disease, as steroid-sparing agents. ${ }^{13}$ Methotrexate has a favorable benefit/risk ratio and evidence of efficacy in $\mathrm{CD},{ }^{22}$ but it has been traditionally less used than thiopurines. However, it should be emphasized that the therapeutic space for conventional monotherapy with immunosuppressants in CD has gradually decreased in recent years. This is due to numerous factors. First, approximately $30 \%$ of patients are refractory to thiopurines, and at least $30 \%$ will be poorly tolerant. In this line, adverse effects may be severe (such as bone marrow suppression, acute pancreatitis, acute hepatitis) or mild (nausea, abdominal pain, flu-like syndrome) but still so annoying as to determine the withdrawal of the treatment. Second, two RCTs showed that azathioprine was not able to modify the main clinical outcomes in patients with early CD, i.e. the temporal phase where there is a window of opportunity to prevent the bowel damage of CD. ${ }^{23,24}$ Third, the use of thiopurines has been clearly associated with an increased risk of malignancies (namely non-melanoma skin cancers, lymphomas - including the rare but fatal hepatosplenic T-cell lymphoma - and urinary tract cancers). ${ }^{25-27}$ Anyway, although immunosuppressants are less used than in the past as monotherapy, they still have a therapeutic role, especially when they are used together with an anti-tumor necrosis factor (TNF) in the so-called combination therapy (see further).

\section{Anti-tumor necrosis factor drugs}

A non-negligible proportion of patients suffer from an aggressive disease, which can be unresponsive to the traditional induction or maintenance therapies, eventually developing a condition of steroid-dependence or even steroid-refractoriness. Therefore, these patients need alternative therapeutic options in order 
to control the disease activity and to avoid surgery. This is the therapeutic space of biological drugs.

The traditional approach of biological therapy in $\mathrm{CD}$ involved the inhibition of mechanisms of adaptive immunity which concur to drive the inflammatory pathway of the disease by blocking the TNF- $\alpha$. The anti-TNFs currently available for the treatment of CD include infliximab - at a dose of $5 \mathrm{mg} / \mathrm{kg}$ at baseline, and after 2 and 6 weeks, and then every 8 weeks adalimumab at a dose of $160 \mathrm{mg}$ at baseline, $80 \mathrm{mg}$ after 2 weeks and then $40 \mathrm{mg}$ every 2 weeks, and certolizumab pegol (available in Switzerland, North America, and a few other countries, but not in Italy). Currently, the use of anti-TNFs in patients with CD is indicated in moderate and/or severe forms of the disease, especially if there is a condition of steroid-dependence or steroid-refractoriness and/or resistance to immunosuppressants. ${ }^{13}$ Furthermore, there are special clinical situations - independently from the luminal activity of the disease - where there is evidence of their efficacy. Indeed, they can be employed in cases of extra-intestinal manifestations, especially for the severe forms of axial or peripheral arthropathy, ${ }^{28}$ and they are the most effective therapy to prevent the postoperative recurrence in patients at high risk for repeated surgery. ${ }^{29}$ In addition, anti-TNFs are indicated for the treatment of complex perianal disease - a hardto-treat clinical setting where the use of advanced therapies, combined with surgery, should be considered very early, according to a clear-cut top-down approach. In particular, infliximab has direct evidence of efficacy in this setting, as it is the only anti-TNF that showed efficacy for the treatment of perianal disease in an ad hoc RCT, ${ }^{30}$ while fistula healing was a secondary endpoint in the CHARM study, the 2007 pivotal phase 3 trial of adalimumab in $\mathrm{CD} .{ }^{31}$ It is worthy to underline that evidence for the above-mentioned special clinical settings is currently not available for both vedolizumab and ustekinumab.

Therapy with anti-TNFs has been shown to be effective in inducing and maintaining remission in patients with $\mathrm{CD}$, in reducing the rates of surgery in patients who are intolerant or unresponsive to traditional therapies, and in determining a significant steroid-sparing effect..$^{32} \mathrm{In}$ addition, their safety profile is acceptable with respect to malignancies, serious infections, and overall mortality, ${ }^{33}$ particularly when they are administered outside the clinical situations where their use is contraindicated (active infections, symptomatic heart failure, demyelinating disorders). ${ }^{34}$ Nonetheless, they are not perfect drugs: the rate of primary non-response is about $20-40 \%$ in RCTs, and about $25 \%$ in observational studies. ${ }^{35}$ Furthermore, a relevant proportion of patients (up to $40 \%$ ) who initially seem to respond to these drugs, subsequently lose the response over time (secondary non-response), mainly due to development of anti-drug antibodies that neutralize their biological activity. ${ }^{36}$ Today, there is the possibility of dosing the serum levels of antiTNF drugs and the presence of anti-drug antibodies (therapeutic drug monitoring) in order to find individual-tailored therapy strategies in case of failure with anti-TNFs. ${ }^{37}$ Anyway, independently of the availability of the laboratory tools for therapeutic drug monitoring, the options to re-gain a clinical benefit in patients with secondary non-response to anti-TNFs include the optimization of the dosage (i.e. the increase of each dose or the reduction of the intervals of administration), the addition of an immunosuppressant (the so-called selective combination therapy), or the switch to a different anti-TNF (Figure 2). Alternatively, the switch to a drug with a different mechanism of action (i.e. vedolizumab or ustekinumab - the socalled swap) may be taken into account. Regarding how to start the treatment with an anti-TNF, a de novo combination therapy (anti-TNF plus an immunosuppressant from the beginning of the biological treatment) is frequently used in several countries to prevent the formation of anti-drug antibodies and improve the clinical outcomes. Although there is evidence that this intensive therapeutic strategy has a slightly higher clinical benefit compared with anti-TNF monotherapy, ${ }^{38}$ there are concerns about the safety of combination therapies, as they increase the risk of severe infections and malignancy. ${ }^{39}$ As a consequence, in order to avoid a potential and dangerous over-treatment, we believe that the combination therapy should be reserved to those patients who develop a secondary non-response to TNF monotherapy (i.e. adding the immunosuppressant at a later time: selective combination therapy), and only after an intensive optimization of the dose of the biologic.

\section{Vedolizumab}

Vedolizumab is a new biologic recently approved for the treatment of adult patients with moderately to severely active $\mathrm{CD}$ who had an inadequate response with, lost response to, or were intolerant to either conventional therapy or anti-TNFs. The efficacy of Vedolizumab in $\mathrm{CD}$ has been demonstrated against placebo in pivotal phase 3 RCTs - GEMINI $2^{40}$ and GEMINI $3^{41}$ - while an open-label, long-term RCT GEMINI LTS - confirmed efficacy of the drug for up to 152 weeks of cumulative treatment. ${ }^{42}$ Vedolizumab is administered intravenously at a fixed dose of 300 $\mathrm{mg}$ at baseline, after 2 and 6 weeks and then every 8 weeks, with the possibility of treatment optimization to every 4 weeks. The drug is a humanized monoclonal antibody that binds the $\alpha 4 \beta 7$ integrin, which is exclusively expressed in the gut, thus selectively blocking the lymphocyte trafficking in the context of the intestinal wall, without systemic interference or 
immunosuppression. This selectivity of action determines an excellent tolerability profile that makes the drug particularly suitable in patients with contraindications to anti-TNF therapy, such as frail or elderly subjects. ${ }^{43}$ This relevant aspect has been clearly confirmed by the cumulative analysis of data from RCTs, which showed how vedolizumab had a favorable safety profile over an extended treatment period. ${ }^{44} \mathrm{On}$ this basis, vedolizumab may be considered for induction and maintenance treatment among steroid-dependent and steroid-refractory patients, along with other biologic agents, and should also be considered a valid second-line treatment in case of primary or secondary failures with anti-TNFs.

\section{Ustekinumab}

In recent years, basic research demonstrated that impaired innate and adaptive immune responses have a relevant role in the pathogenesis of the intestinal damage in CD, with interleukin (IL)-12 and IL-23 acting as key drivers of the adaptive response. ${ }^{45}$ As a consequence, blockers of IL-12/23 and selective blockers of IL-23 have been investigated as therapeutic options in CD. In this line, ustekinumab - a fully-humanized IgG1k monoclonal antibody that binds to the $\mathrm{p} 40$ subunit of both IL-12 and IL-23, and that was already used in psoriasis ${ }^{46,47}$ and psoriatic arthritis ${ }^{48,49}$ - has been re- cently approved for the treatment of $\mathrm{CD}$ following the good efficacy demonstrated in the Phase 3 trial program, called UNITI. ${ }^{50}$ It is administered with a single, weight-based, intravenous dose of induction, followed by subcutaneous $90 \mathrm{mg}$ injections every 8 weeks. In particular, the drug has been approved for the treatment of patients with moderate-severe $\mathrm{CD}$ who had an inadequate response with, lost response to, or were intolerant to either conventional therapy or TNF- $\alpha$ inhibitors, as second-line therapy. Theoretically, ustekinumab could also be considered as a first-line agent - although its high cost represents a significant issue - especially in frail patients, due to its favorable safety profile. Indeed, there is extensive knowledge of the safety of this drug in the field of dermatology and rheumatology: data from the PSOLAR - a long-term registry of more than 12,000 patients with psoriasis treated with different biologics $^{51}$ - showed that patients receiving ustekinumab had the lowest rate of serious infections compared with the other biologics used in this disease. This favorable safety profile has been confirmed by the long-term extension of IM-UNITI ${ }^{52}$ In addition, its use can be considered in special situations, such as in case of paradoxical inflammation of skin or joints under therapy with anti-TNFs, in case of concomitant dermatological or rheumatological diseases, or in case of extra-intestinal manifestations, where it could be pre-

\section{Loss of response to Anti-TNF monotherapy}

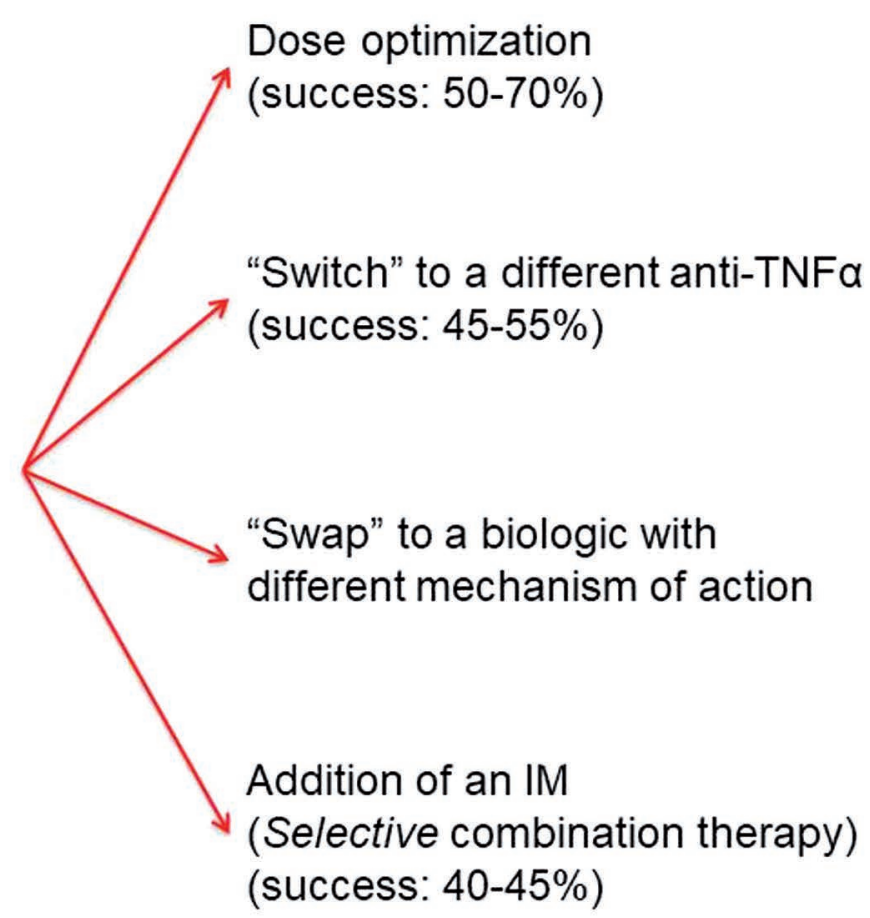

Figure 2. Therapeutic options to re-achieve a clinical benefit in case of secondary non-response to monotherapy with anti-tumor necrosis factors (TNFs). IM, immunosuppressant. 
ferred over vedolizumab due to its presumed systemic anti-inflammatory effects.

\section{Darvadstrocel}

The most recent therapeutic option in CD - specifically for the treatment of complex perianal disease is darvadstrocel, a preparation of expanded human allogeneic adult mesenchymal stem cells extracted from adipose tissue. This advanced therapy is designed for intralesional use under anesthesia, during surgery for perianal disease. In particular, darvadstrocel is indicated for the treatment of complex perianal fistulas in adult patients with non-active or mildly active luminal $\mathrm{CD}$, in cases of inadequate response to at least one conventional or biological therapy. The treatment was approved following the good results obtained by the pivotal Phase 3 study ADMIRE-CD, ${ }^{53}$ where a single dose of darvadstrocel in the surrounding perianal fistula tissue - after fistula treatment with standard care - was significantly more effective than placebo in terms of closure of the fistula plus absence of abscesses evaluated by magnetic resonance at 24 weeks. As a consequence, darvadstrocel should be considered a promising therapy for refractory complex perianal disease, a difficult-to-treat and highly disabling clinical setting.

\section{Future perspectives: novel biologics and small molecule drugs}

Over the past 20 years, drug research in IBD has mainly focused on the development of new biologics. As a consequence, several anti-TNFs monoclonal antibodies and, more recently, vedolizumab and ustekinumab have become available in clinical practice, while other biologics are currently under development. However, monoclonal antibodies have limitations. In fact, they can be administered only parenterally, and they are not universally effective, mainly due to the fact that a significant percentage of patients develop a loss of response over time that is often caused by immunogenic factors (i.e. formation of anti-drug antibodies). ${ }^{36}$ Given these premises, there is now a growing interest in new, orally administered, small molecule drugs (SMDs), compounds that are chemically different from biologics. ${ }^{54} \mathrm{In}$ deed, SMDs have a very low molecular weight $(<1$ $\mathrm{kDa}$ ), and this allows oral administration and the direct interaction with intracellular targets. Furthermore, they have no immunogenicity issues. Finally, the manufacturing process of SMDs is based on chemical synthesis, thus it is much simpler than that of biologics.

Based on these assumptions, the future therapeutic armamentarium for $\mathrm{CD}$ will be enriched with both new biologics and SMDs. Focusing only on the molecules at the most advanced phases of development, we should mention - among the new biologics - the selective IL-23 inhibitors brazikumab, ${ }^{55}$ risankizumab ${ }^{56,57}$ and guselkumab. These drugs - unlike ustekinumab - bind only the p19 subunit of IL23 , producing a specific inhibition of IL-23. It has been hypothesized that this more restricted target could increase safety, allowing a normal IL-12-mediated Th1 response against pathogens and tumor immune surveillance. ${ }^{58}$ Another group of biologics under development includes novel anti-integrins, drugs that are intended to be more effective than vedolizumab, preserving at the same time the gut selectivity. Etrolizumab is a dual-action anti-integrin antibody which has demonstrated endoscopic and symptomatic improvements in patients with moderate to severe active $\mathrm{CD}$ in the BERGAMOT Phase 3 trial. ${ }^{59}$ SHP-647 is a monoclonal antibody that addresses MAdCAM-1 and that has shown interesting, though not exciting, results from the Phase 2 trials in moderate to severe $\mathrm{CD} .{ }^{60,61}$ Focusing on SMDs under development for $\mathrm{CD}$, ozanimod is a new drug that selectively modulates the sphingosine-1-phosphate (S1P) receptor. S1P has an important role in the pathways of homing of the lymphocytes into the lymphoid organs, and in their migration in the circulation. Ozanimod prevents the lymphocytes from leaving lymphoid tissues, and this trapping leads to a reduction in circulating effector $\mathrm{T}$ cells, with consequent selective suppression of the immune system in the absence of a strong overall immunosuppressive effect. ${ }^{62}$ In a recent Phase 2 double-blind RCT in ulcerative colitis (TOUCHSTONE), Ozanimod showed good efficacy with acceptable tolerability and safety, ${ }^{63}$ while its efficacy in CD is currently under evaluation. ${ }^{64}$ Following the excellent efficacy demonstrated by tofacitinib - a non-selective JAK inhibitor - in the Phase 3 trial program for ulcerative colitis (OCTAVE), ${ }^{65}$ other JAK inhibitors are currently being assessed for $\mathrm{CD}$. In particular, the research is focusing on selective JAK inhibitors, as there are safety concerns for tofacitinib. In fact, the JAK-STAT pathway is used by several cytokines to exert their pro-inflammatory effects, so the simultaneous block of JAK1, JAK2, and JAK3 induced by tofacitinib translates into high-level immunosuppression. In this regard, filgotinib is a selective JAK 1 inhibitor which showed interesting results in the Phase 2 study for CD (FITZROY), ${ }^{66}$ particularly among patients who were anti-TNF-naive, while a Phase 3 study (DIVERSITY) is currently in progress ${ }^{67} \mathrm{An}-$ other highly promising selective JAK1 inhibitor is upadacitinib, whose safety and efficacy as induction therapy has recently been demonstrated in patients with $\mathrm{CD}$ in a phase 2 study (CELEST). ${ }^{68}$ 


\section{References}

1. Abraham $\mathrm{C}$, Cho JH. Inflammatory bowel disease. $\mathrm{N}$ Engl J Med 2009;361:2066-78.

2. Pariente B, Cosnes J, Danese S, et al. Development of the Crohn's disease digestive damage score, the Lémann score. Inflamm Bowel Dis 2011;17:1415-22.

3. Cosnes J, Bourrier A, Nion-Larmurier I, et al. Factors affecting outcomes in Crohn's disease over 15 years. Gut 2012;61:1140-5.

4. Hold GL, Smith M, Grange C, et al. Role of the gut microbiota in inflammatory bowel disease pathogenesis: what have we learnt in the past 10 years? World J Gastroenterol 2014;20:1192-210.

5. Veber DF, Johnson SR, Cheng HY, et al. Molecular properties that influence the oral bioavailability of drug candidates. J Med Chem 2002;45:2615-23.

6. Olivera P, Danese S, Peyrin-Biroulet L. Next generation of small molecules in inflammatory bowel disease. Gut 2017;66:199-209.

7. Torres J, Mehandru S, Colombel JF, et al. Crohn's disease. Lancet. 2017;389:1741-55.

8. Shah SC, Colombel JF, Sands BE, et al. Systematic review with meta-analysis: mucosal healing is associated with improved long-term outcomes in Crohn's disease. Aliment Pharmacol Ther 2016;43:317-33.

9. Colombel JF, Reinisch W, Mantzaris GJ, et al. Randomised clinical trial: deep remission in biologic and immunomodulator naïve patients with Crohn's disease - a SONIC post hoc analysis. Aliment Pharmacol Ther 2015;41:734-46.

10. Khanna R, Bressler B, Levesque BG, et al. Early combined immunosuppression for the management of Crohn's disease (REACT): a cluster randomised controlled trial. Lancet 2015;386:1825-34.

11. Mosli MH, Zou G, Garg SK, et al. C-reactive protein, fecal calprotectin, and stool lactoferrin for detection of endoscopic activity in symptomatic inflammatory bowel disease patients: a systematic review and meta-analysis. Am J Gastroenterol 2015;110:802-19.

12. Colombel JF, Panaccione R, Bossuyt P, et al. Effect of tight control management on Crohn's disease (CALM): a multicentre, randomised, controlled phase 3 trial. Lancet 2018;390:2779-89.

13. Gomollón F, Dignass A, Annese V, et al. 3rd European evidence-based consensus on the diagnosis and management of Crohn's Disease 2016: Part 1: diagnosis and medical management. J Crohns Colitis 2017;11:3-25.

14. Tremaine WJ, Schroeder KW, Harrison JM, et al. A randomized, double-blind, placebo-controlled trial of the oral mesalamine (5-ASA) preparation, Asacol, in the treatment of symptomatic Crohn's colitis and ileocolitis. J Clin Gastroenterol 1994;19:278-82.

15. Singleton JW, Hanauer SB, Gitnick GL, et al. Mesalamine capsules for the treatment of active Crohn's disease: results of a 16-week trial. Pentasa Crohn's Disease Study Group. Gastroenterology 1993;104:1293301 .

16. Malchow H, Ewe K, Brandes JW, et al. European Cooperative Crohn's Disease Study (ECCDS): results of drug treatment. Gastroenterology 1984;86:249-66.

17. Feagan BG. 5-ASA therapy for active Crohn's disease: old friends, old data, and a new conclusion. Clin Gastroenterol Hepatol 2004;2:376-8.

18. Thomsen OO, Cortot A, Jewell D, et al. A comparison of budesonide and mesalamine for active Crohn's disease. International Budesonide-Mesalamine Study Group. N Engl J Med 1998;339:370-4.

19. Ford AC, Khan KJ, Talley NJ, et al. 5-aminosalicylates prevent relapse of Crohn's disease after surgically induced remission: systematic review and meta-analysis. Am J Gastroenterol 2011;106:413-20.

20. Rezaie A, Kuenzig ME, Benchimol EI, et al. Budesonide for induction of remission in Crohn's disease. Cochrane Database Syst Rev 2015; 6:CD000296.

21. Faubion WA, Loftus EV, Harmsen WS, et al. The natural history of corticosteroid therapy for inflammatory bowel disease: a population-based study. Gastroenterology 2001;121:255-60.

22. McDonald JW, Wang Y, Tsoulis DJ, et al. Methotrexate for induction of remission in refractory Crohn's disease. Cochrane Database Syst Rev 2014;8:CD003459.

23. Cosnes J, Bourrier A, Laharie D, et al. Early administration of azathioprine vs conventional management of Crohn's disease: a randomized controlled trial. Gastroenterology 2013;145:758-65.

24. Panés J, López-Sanromán A, Bermejo F, et al. Early azathioprine therapy is no more effective than placebo for newly diagnosed Crohn's disease. Gastroenterology 2013;145:766-74.

25. Beaugerie L, Brousse N, Bouvier AM, et al. Lymphoproliferative disorders in patients receiving thiopurines for inflammatory bowel disease: a prospective observational cohort study. Lancet 2009;374:1617-25.

26. Bourrier A, Carrat F, Colombel JF, et al. Excess risk of urinary trac cancers in patients receiving thiopurines for inflammatory bowel disease: a prospective observational cohort study. Aliment Pharmacol Ther 2016;43:252-61.

27. Peyrin-Biroulet L, Khosrotehrani K, Carrat F, et al. Increased risk for non-melanoma skin cancers in patients who receive thiopurines for inflammatory bowel disease. Gastroenterology 2011;141:1621-8.

28. Gionchetti P, Dignass A, Danese S, et al. 3rd European evidence-based consensus on the diagnosis and management of crohn's disease 2016: Part 2: surgical management and special situations. J Crohns Colitis 2017;11:135-49.

29. Nguyen V, Kanth R, Gazo J, et al. Management of postoperative Crohn's disease in 2017: where do we go from here? Exp Rev Gastroenterol Hepatol 2016;10:1257-69.

30. Sands BE, Anderson FH, Bernstein CN, et al. Infliximab maintenance therapy for fistulizing Crohn's disease. N Engl J Med 2004;350:876-85.

31. Colombel JF, Schwartz DA, Sandborn WJ, et al. Adalimumab for the treatment of fistulas in patients with Crohn's disease. Gut 2009;58:940-8.

32. Lawson MM, Thomas AG, Akobeng AK. Tumour necrosis factor alpha blocking agents for induction of remission in ulcerative colitis. Cochrane Database Syst Rev 2006;19:CD005112.

33. Bonovas S, Fiorino G, Allocca M, et al. Biologic therapies and risk of infection and malignancy in patients with inflammatory bowel disease: a systematic review and network meta-analysis. Clin Gastroenterol Hepatol 2016;14:1385-97. 
34. Peyrin-Biroulet L. Anti-TNF therapy in inflammatory bowel diseases: a huge review. Minerva Gastroenterol Dietol 2010;56:233-43.

35. Ford AC, Sandborn WJ, Khan KJ, et al. Efficacy of biological therapies in inflammatory bowel disease. Systematic review and meta-analysis. Am J Gastroenterol 2011;106:644-59.

36. Ben-Horin S, Chowers Y. Review article: loss of response to anti-TNF treatments in Crohn's disease. Aliment Pharmacol Ther 2011;33:987-95.

37. Seinen ML, De Boer NK, van Bodegraven AA. Key insights from therapeutic drug monitoring in Crohn's disease patients. Exp Opin Drug Metab Toxicol 2019;15:399-406.

38. Colombel JF, Sandborn WJ, Reinisch W, et al. Infliximab, azathioprine, or combination therapy for Crohn's disease. N Engl J Med 2010;362:1383-95.

39. Dulai PS, Siegel CA, Colombel JF, et al. Systematic review: monotherapy with antitumour necrosis factor $\alpha$ agents versus combination therapy with an immunosuppressive for IBD. Gut 2014;63:1843-53.

40. Sandborn WJ, Feagan BG, Rutgeerts P, et al. Vedolizumab as induction and maintenance therapy for Crohn's disease. N Engl J Med 2013;369:711-21.

41. Sands BE, Feagan BG, Rutgeerts P, et al. Effects of vedolizumab induction therapy for patients with Crohn's disease in whom tumor necrosis factor antagonist treatment failed. Gastroenterology 2014;147:618-27.

42. Vermeire S, Loftus EV Jr, Colombel JF, et al. Long-term efficacy of vedolizumab for Crohn's disease. J Crohns Colitis 2017;11:412-24.

43. Wang MC, Zhang LY, Han W, et al. PRISMA-efficacy and safety of vedolizumab for inflammatory bowel diseases: a systematic review and meta-analysis of randomized controlled trials. Medicine 2014;93:e326.

44. Colombel JF, Sands BE, Rutgeerts P, et al. The safety of vedolizumab for ulcerative colitis and Crohn's disease. Gut. 2017;66:839-51.

45. Xavier RJ, Podolsky DK. Unravelling the pathogenesis of inflammatory bowel disease. Nature 2007;448:427-34.

46. Leonardi CL, Kimball AB, Papp KA, et al. Efficacy and safety of ustekinumab, a human interleukin-12/23 monoclonal antibody, in patients with psoriasis: 76-week results from a randomised, double-blind, placebo-controlled trial (PHOENIX 1). Lancet 2008;371:1665-74.

47. Papp KA, Langley RG, Lebwohl M, et al. Efficacy and safety of ustekinumab, a human interleukin-12/23 monoclonal antibody, in patients with psoriasis: 52-week results from a randomised, double-blind, placebo-controlled trial (PHOENIX 2). Lancet 2008;371:1675-84.

48. McInnes IB, Kavanaugh A, Gottlieb AB, et al. Efficacy and safety of ustekinumab in patients with active psoriatic arthritis: 1 year results of the phase 3, multicentre, double-blind, placebo-controlled PSUMMIT 1 trial. Lancet 2013;382780-9.

49. Ritchlin C, Rahman P, Kavanaugh A, et al. Efficacy and safety of the anti-IL-12/23 p40 monoclonal antibody, ustekinumab, in patients with active psoriatic arthritis despite conventional non-biological and biological antitumour necrosis factor therapy: 6-month and 1-year results of the phase 3, multicentre, double-blind, placebo-controlled, randomised PSUMMIT 2 trial. Ann Rheum Dis 2014;73:990-9.
50. Feagan BG, Sandborn WJ, Gasink C, et al. Ustekinumab as induction and maintenance therapy for Crohn's disease. N Engl J Med 2016;375:1946-60.

51. Papp K, Gottlieb AB, Naldi L, et al. Safety surveillance for ustekinumab and other psoriasis treatments from the psoriasis longitudinal assessment and registry (PSOLAR). J Drugs Dermatol 2015;14:706-14.

52. Sandborn WJ, Rutgeerts P, Gasink C, et al. Long-term efficacy and safety of ustekinumab for Crohn's disease through the second year of therapy. Aliment Pharmacol Ther 2018;48:65-77.

53. Panés J, García-Olmo D, Van Assche G, et al. Expanded allogeneic adipose-derived mesenchymal stem cells (Cx601) for complex perianal fistulas in Crohn's disease: a phase 3 randomised, double-blind controlled trial. Lancet 2016;388:1281-90.

54. Olivera P, Danese S, Peyrin-Biroulet L. Next generation of small molecules in inflammatory bowel disease. Gut 2017;66:199-209.

55. Sands BE, Chen J, Feagan BG, et al. Efficacy and safety of MEDI2070, an antibody against interleukin 23, in patients with moderate to severe Crohn's disease: a phase 2a study. Gastroenterology 2017;153:77-86.

56. Feagan BG, Sandborn WJ, D'Haens G, et al. Induction therapy with the selective interleukin-23 inhibitor risankizumab in patients with moderate-to-severe Crohn's disease: a randomised, double-blind, placebocontrolled phase 2 study. Lancet 2017;389:1699-709.

57. Feagan BG, Panés J, Ferrante M, et al. Risankizumab in patients with moderate to severe Crohn's disease: an open-label extension study. Lancet Gastroenterol Hepatol 2018 [Epub ahead of print].

58. Croxford AL, Kulig P, Becher B. IL-12-and IL-23 in health and disease. Cytokine Growth Factor Rev 2014;25:415-21

59. ClinicalTrials.gov. A study to assess whether etrolizumab is a safe and effective treatment for participants with moderately to severely active Crohn's disease (CD). NCT02394028. April 2, 2018. Available from: https://clinicaltrials.gov/ct2/show/NCT02394028 Accessed: June 10, 2018.

60. Sandborn WJ, Lee SD, Tarabar D, et al. Phase II evaluation of anti-MAdCAM antibody PF-00547659 in the treatment of Crohn's disease: report of the OPERA study. Gut 2018;67:1824-35.

61. ClinicalTrials.gov. A study to monitor long-term treatment with PF-00547659 (OPERA II). NCT01298492. Available from: https://clinicaltrials.gov/ct2/show/study/ NCT01298492 Accessed: June 10, 2019.

62. Degagné E, Saba JD. Slpping fire: Sphingosine-1-phosphate signaling as an emerging target in inflammatory bowel disease and colitis-associated cancer. Clin Exp Gastroenterol 2014;7:205-14.

63. Sandborn WJ, Feagan BG, Wolf DC, et al. Ozanimod induction and maintenance treatment for ulcerative colitis. N Engl J Med 2016;374:1754-62.

64. ClinicalTrials.gov. Efficacy and Safety Trial of RPC1063 for Moderate to Severe Crohn's Disease. NCT02531113. Available from: https://clinicaltrials.gov/ct2/show/ NCT02531113 Accessed: June 10, 2019.

65. Sandborn WJ, Su C, Sands BE, et al. Tofacitinib as induction and maintenance therapy for ulcerative colitis. N Engl J Med 2017;376:1723-36. 
66. Vermeire S, Schreiber S, Petryka R, et al. Clinical remission in patients with moderate-to-severe Crohn's disease treated with filgotinib (the FITZROY study): results from a phase 2, double-blind, randomized, placebo-controlled trial. Lancet 2017;389:266-75.

67. ClinicalTrials.gov. Filgotinib in the induction and maintenance of remission in adults with moderately to severely active Crohn's disease (Diversity1). NCT02914561. June 10, 2019. Available from: https://clinicaltrials.gov/ct2/ show/NCT02914561

68. Sandborn WJ, Feagan BG, Sands BE, et al. Safety and efficacy of ABT-494 (Upadacitinib), an oral Jak1 inhibitor, as induction therapy in patients with Crohn's disease: results from celest. Gastroenterology 2017;152:S1308-9. 